\title{
Tailoring ultrafast singlet fission by structural modification of phenazinothiadiazoles
}

\author{
N. Alagna ${ }^{1}$, J. Han³ ${ }^{3}$ J. Herz ${ }^{1}$, J. L. Perez Lustres¹, S. Hahn², S. Koser², Florian L. Geyer², Sebastian \\ Hahn $^{2}$, U. Bunz ${ }^{2}$, A. Dreuw ${ }^{3}$, T. Buckup ${ }^{1}$, M. Motzkus ${ }^{1}$ \\ ${ }^{1}$ Physikalisch-Chemisches Institut, Ruprecht-Karls Universität Heidelberg, D-69210, Heidelberg, Germany \\ ${ }^{2}$ Organisch-Chemisches Institut, Ruprecht-Karls Universität Heidelberg, D-69210, Heidelberg, Germany \\ ${ }^{3}$ Institut für Wissenschaftliches Rechnen, Ruprecht-Karls Universität Heidelberg, D-69210, Heidelberg, Germany
}

\begin{abstract}
Ultrafast transient absorption and quantum chemistry calculations are combined to demonstrate singlet fission in newly functionalized TIPS-Tetracenes. The coupling strength (but not the energy gap) between $\mathrm{S}_{1}$ and ${ }^{1}(\mathrm{TT})$ states gauges singlet fission efficiency and rate.
\end{abstract}

\section{Introduction}

The efficiency limit of organic photovoltaic devices can be overcome by Singlet Fission (SF), a photoinduced processes where two triplet states result from the "fission" of one excited singlet state [1]. The process was observed in films and occurs on the fs timescale with high yields. Molecular structure, and packing arrangement, are critical for SF. For instance, the SF rate was found to be increased by a factor of two by double Nitrogen substitution in the aromatic skeleton of TIPS-Pentacene (TIPS-Pn) [2]. In turn, further Nitrogen substitution slows down the SF rate [3]. In an attempt to rationalise this effect, a series of phenazinothiadiazoles has been synthetized. The molecules, with four fused aromatic rings, bear some resemblance with TIPS-tetracene, where SF is endothermic [4]. The antracenothiadiazole core meets the key requirement for SF, namely, that the energy of the first excited singlet state exceeds twice the energy of the lowest triplet, $\mathrm{E}\left(\mathrm{S}_{1}\right)>2 \mathrm{E}\left(\mathrm{T}_{1}\right)$. This is shown by quantum chemical calculations, which suggest minor changes upon halogen substitution. Ultrafast broadband transient absorption provides experimental evidence and supports the correlation between key molecular properties and SF rates.

\section{Experimental details}

Broadband transient absorption (TA) is performed with fs pump pulses and supercontinuum probing at $1 \mathrm{kHz}$ repetition rate. Pump pulses $(<15 \mathrm{fs})$ spectrally centred at $620 \mathrm{~nm}$ are generated in a home-built non-collinear optical parameter amplifier. The supercontinuum white light ranges from 450 to $720 \mathrm{~nm}$ and is generated in a $1 \mathrm{~mm}$ sapphire plate. A time delayed white light laser (STM-2-UV) is used as probe 
for sub- $\mu$ s measurements. The synthesis of molecules here studied (TDT, $\mathrm{TDCl}_{4}$ and $\mathrm{TDF}_{4}$, see Fig. 1a) was described elsewhere [5]. Thin films were prepared by spin coating. The layers are typically $40 \mathrm{~nm}$ thick. UV-Vis absorption spectra of the films are shown in Fig. $1 \mathrm{~b}$.

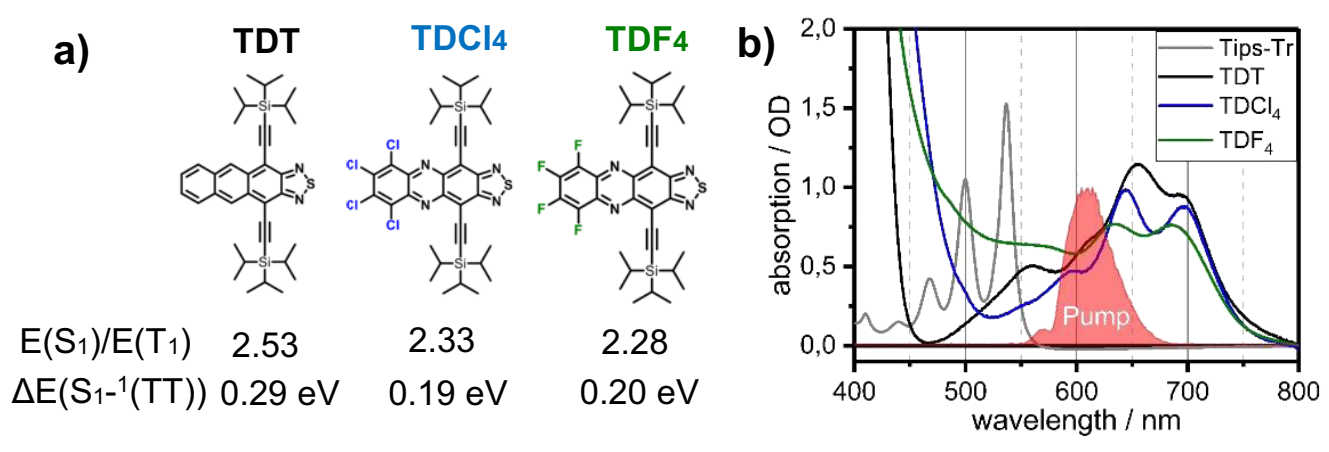

Fig. 1. a) Molecular structures of TIPS-anthracenothiadiazole (TDT) and its tetrachloro $\left(\mathrm{TDCl}_{4}\right)$ and tetrafluoro $\left(\mathrm{TDF}_{4}\right)$ derivatives; calculated $\mathrm{S}_{1}{ }^{-1}(\mathrm{TT})$ vertical energy gaps and energy ratios $\mathrm{E}(\mathrm{S} 1) / \mathrm{E}(\mathrm{T} 1)$ are indicated; b) Absorption spectra TDT (black), $\mathrm{TDCl}_{4}$ (blue), $\mathrm{TDF}_{4}$ (green) and TIPS-Tetracene (TIPS-Tn, grey) in thin film (left) and in toluene solution (right). Typical pump spectrum is shown as a red-filled curve.

\section{Results and discussion}

The transient absorption spectra of $\mathrm{TDCl}_{4}$ in thin film evolves on the fs timescale. A broad excited state absorption (ESA) band with maximum at $560 \mathrm{~nm}$ is observed at earliest time. The band decays on the way towards longer delays while a second ESA band rises concomitantly at $620 \mathrm{~nm}$ (Fig 2a). Sharply defined isosbestic points are observed at 480, 570 and $640 \mathrm{~nm}$. Single exponential fits yield characteristic times of $(370 \pm 10)$ fs for the decay of ESA at $560 \mathrm{~nm}$ and $(360 \pm 12)$ fs for the rise of the new ESA band at $620 \mathrm{~nm}$ (Fig 2b). No further spectral evolution is observed. The resulting band-shape stays constant and decays with a much longer time constant of $(180 \pm 30)$ ns, which is typical for triplet decay (Fig. 3a). Thus, the slowly decaying spectrum is assigned to the triplet state, which is formed by SF with a characteristic time constant of $\tau_{\mathrm{SF}}\left(\mathrm{TDCl}_{4}\right)=360$ fs. The spectral assignment was confirmed by quantum chemical calculations (see below). TDT and $\mathrm{TDF}_{4}$ show very similar spectral evolution. The corresponding SF time constants are $\tau_{\mathrm{SF}}(\mathrm{TDT})=90$ and $\tau_{\mathrm{SF}}\left(\mathrm{TDF}_{4}\right)=300 \mathrm{fs}$.

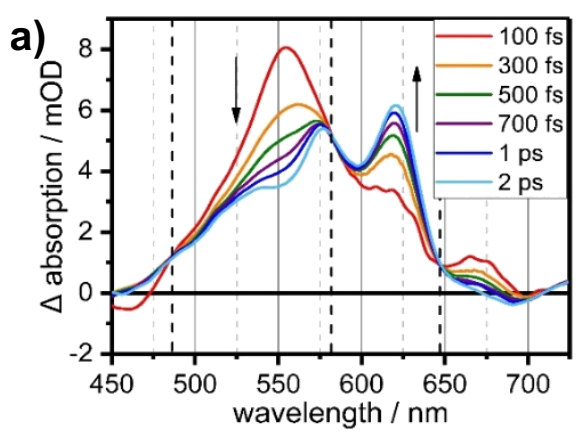

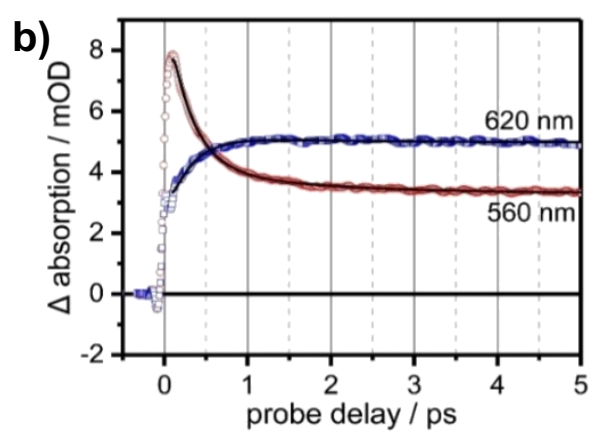


Fig. 2. a) Transient absorption spectra of $\mathrm{TDCl}_{4}$ at the indicated pump-probe delays and b) Kinetic traces obtained at $560 \mathrm{~nm}$ (red circles) and $620 \mathrm{~nm}$ (blue squares). Monoexponential fits are shown in black.

The behaviour just described can be rationalized with the help of quantum chemical calculations at the B3LYP/6-31G(d) level of theory. Vertical excitation energies and transient spectra of the $\mathrm{S}_{1}$ state, the ${ }^{1}(\mathrm{TT})$ intermediate and the final $\mathrm{T}_{1}$ state were obtained (Figs. 1a and $3 \mathrm{~b})$. All molecules fulfil the condition for $\mathrm{SF}\left(\mathrm{E}\left(\mathrm{S}_{1}\right)>2 \mathrm{E}\left(\mathrm{T}_{1}\right)\right)$. In comparison, $\mathrm{E}\left(\mathrm{S}_{1}\right)$ is calculated to be 1.99 times $\mathrm{E}\left(\mathrm{T}_{1}\right)$ in TIPS-Tetracene. Moreover, calculated triplet-triplet absorption spectra show qualitative agreement with the nondecaying component of the fs transient absorption experiment at 5 ps delay (Fig. 3b), which further confirms ultrafast triplet formation by SF.

The calculated singlet-triplet energy gap of $\mathrm{TDF}_{4}$ and TDT differs by $5 \%$ only, while the experimental SF rate changes by a factor of 4 in these compounds. These changes in the experimental SF rates cannot either be explained by the calculated energy gaps between the $S_{1}$ state and the ${ }^{1}(\mathrm{TT})$, since smaller energy gaps are expected to lead to faster SF rates. The contrary is observed, e.g. TDT shows the larger energy gap, but the faster SF among all compounds. This suggests that it is the coupling strength between the $S_{1}$ and the ${ }^{1}(\mathrm{TT})$ states (and not the vertical energy gap) that plays a major role in determining the singlet fission rate for these new compounds.
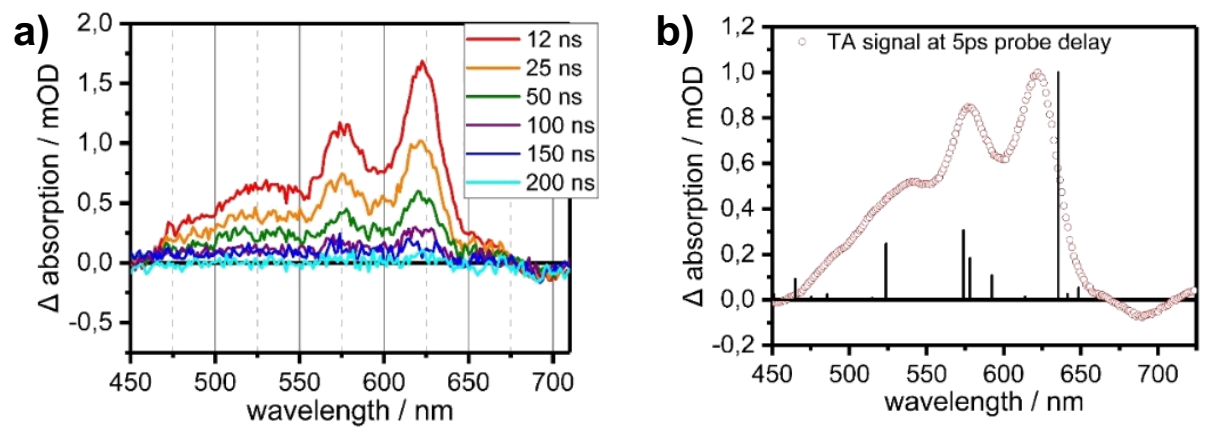

Fig. 3 a) Time evolution of the $\mathrm{TDCl}_{4}$ transient absorption spectrum in the ns range. b) Transient absorption spectrum of $\mathrm{TDCl}_{4}$ at 5 ps delay (red circles). Triplet-triplet optical transitions calculated with TD-DFT/B3LYP/6-31G(d) are shown as black vertical bars. Amplitudes are proportional to the corresponding oscillator strengths.

\section{References}

1. M.B. Smith and J. Michl, Chem. Rev., 110, 6891 (2010)

2. J. Herz, T. Buckup, F. Paulus, J. Engelhart,U. H. F. Bunz and M. Motzkus, J. Phys. Chem. Lett., 5, 2425 (2014)

3. J. Herz, T. Buckup, F. Paulus, J. U. Engelhart, U. H. F. Bunz and M Motzkus, J. Phys. Chem. , 119, 6602 (2015)

4. H. L. Stern, A. J. Musser, S. Gelinas, P. Parkinson, L. M. Herz, M. J. Bruzek, J. Anthony, R. H. Friend and B. J. Walker, PNAS, 112, 7656 (2015)

5. B. D. Lindner, F. Paulus, A. L. Appleton, M. Schaffroth, J. U. Englehart, K. M. Schelkle, O. Tverskoy, F. Rominger, M. Hambuerger and U. H. F. Bunz, J. Mater. Chem. C, 2, 2014 